\title{
Dynamics of Ions of Intact Proteins in the Orbitrap Mass Analyzer
}

\author{
Alexander Makarov and Eduard Denisov \\ Thermo Fisher Scientific, Bremen, Germany
}

While allowing analysis of intact proteins without a theoretical upper mass limit, the Orbitrap mass analyzer demonstrates reduced resolving power as ion mass increases even at a constant mass-to-charge ratio. It is shown that this effect comes from the effects of ion scattering on background gas molecules. The main mechanisms causing decay of acquired transient appear to be fragmentation as well as accelerated dephasing of ion packets. Isotopic resolution of proteins including bovine serum albumin (MW $66.4 \mathrm{kDa}$ ) and transferrin (MW $78 \mathrm{kDa}$ ) has also been demonstrated. As a part of this study, detection of individual multiply-charged ions of myoglobin (MW $16.9 \mathrm{kDa}$ ) has been demonstrated. Quantized distribution of signal intensities for +20 myoglobin ions well above the noise threshold was observed, with high mass accuracy and resolution of recorded individual ions used as an independent confirmation of correct assignment of signal to ions rather than to noise. The latter also allowed us to benchmark the sensitivity of image-current detection and explore in detail factors responsible for signal decay. (J Am Soc Mass Spectrom 2009, 20, 1486-1495) (C 2009 Published by Elsevier Inc. on behalf of American Society for Mass Spectrometry

$\mathrm{F}$ ourier transform mass spectrometry (FTMS) [1] offers several advantages for the analysis of biological samples, including high mass resolution, high mass accuracy, high sensitivity, and wide mass range. At a first glance, very high resolving power typical for FTMS, in excess of $10^{5}-10^{6}$, in combination with high charge state characteristic for electrospray ionization (ESI) promises an easy methodology for resolving isotopic structure even for heavier proteins. In reality, issues of sample preparation and separation, space charge limitations, splitting of signal into a multitude of isotopes and isoforms, scattering on residual gas, and other practical limitations make such analysis of intact proteins a non-trivial task. For example, in FT ICR mass spectrometry the heaviest protein resolved so far with unit (isotopic) resolution has a molecular mass of ca. $115 \mathrm{kDa}$ (truncated cardiac myosin binding protein C, cMyBP-C [2].

Another member of the FTMS family, the Orbitrap mass analyzer, provides $m / z$-independent trapping conditions and thus in theory allows analysis of intact proteins without upper mass limit. Application of this analyzer to mass measurement of intact proteins is exemplified in $[3,4]$. However, its application to resolving isotopic distributions has only been shown on routine basis for small proteins up to around $30 \mathrm{kDa}$ [5]. This work is devoted to investigations of reasons for such a limitation and exploration of possible ways to extend this upper mass limit.

Address reprint requests to Dr. Alexander A. Makarov, Thermo Fisher Scientific (Bremen) GmbH, Hanna-Kunath Str. 11, 28199 Bremen, Germany. E-mail: alexander.makarov@thermofisher.com

\section{Theoretical}

Transients of Protein Ions in the Orbitrap Mass Analyzer

As previously demonstrated in [6], time domain signals from multiply-charged biopolymer ions exhibit characteristic and predictable beat patterns due to the phase relationship and constructive/destructive nature of the interference between the signals arising from various isotope and charge state populations. Useful frequency information in these time domain signals is extracted only from equally spaced "beats" while time span between these beats is essentially lost for signal acquisition. The physical reason for this is the fact that between beats the ion ensemble is widely dispersed due to slightly different frequencies of packets within the isotopic envelope and thus produces no net signal at the detection electrodes.

Following [6], let us consider isotopic beat patterns for the particular case of the Orbitrap mass analyzer and start from the general equation for the frequency of axial oscillations in this analyzer:

$$
f=\frac{1}{2 \pi} \sqrt{\frac{e}{(m / z)} \cdot k}
$$

where $e$ is the elementary charge $\left(1.602 \times 10^{-19} \mathrm{C}\right)$ and $k$ is a characteristic constant of the field. We can estimate the frequency splitting between adjacent isotope peaks by subtracting frequencies of mass peaks with mass numbers $M$ and $(M+\Delta M)$ and the same charge state $z$ : 


$$
\begin{aligned}
\Delta f=\frac{1}{2 \pi} & \sqrt{\frac{e}{\left((M+\Delta M) m_{0} / z\right)} \cdot k} \\
& -\frac{1}{2 \pi} \sqrt{\frac{e}{\left(M m_{0} / z\right)} \cdot k} \approx-\frac{f}{2} \cdot \frac{\Delta M}{M}
\end{aligned}
$$

where frequency $f$ is calculated for mass number $M$ and we have used an approximation:

$$
\sqrt{\frac{M}{M+\Delta M}} \approx 1-\frac{\Delta M}{2 \cdot M}
$$

For isotopic peaks separated by one atomic mass unit (1.003 $\mathrm{u}$ is typical for proteins and peptides), eq 2 could be further simplified to:

$$
\Delta f_{1} \approx-\frac{f}{2} \cdot \frac{1}{M}
$$

According to [6], this makes it possible to estimate the period of beat patterns:

$$
\Delta t_{i}=1 /\left|\Delta f_{1}\right| \approx \frac{2 M}{f}
$$

i.e., it grows as $\mathrm{M}^{3 / 2} / \mathrm{z}^{1 / 2}$. For a commercial LTQ Orbitrap instrument, eq 5 could be rewritten as:

$$
\Delta t_{i}(s) \approx 2.9 \cdot 10^{-7} \cdot[M(D a)]^{3 / 2} / z^{1 / 2}
$$

Equation 2 can also be used to link the characteristic width of beats in time domain [6] with the width of the isotope distribution $\Delta M_{w}$ :

$$
\Delta t_{w}=1 /|\Delta f| \approx \frac{2 \cdot K}{f} \cdot \frac{M}{\Delta M_{w}}
$$

where $\mathrm{K}$ is a constant of order of magnitude near unity, whose exact value depends on the functional form of the envelope of the isotope distribution and the particular criteria used to define peak widths.

To resolve isotope peaks within a single charge state, a minimum of two isotopic beats must be observed [6]. Coherence of ion packets in the Orbitrap analyzer is provided by injecting them as short packets at an axial coordinate near the turning point. This also means that they all start oscillating at about the same phase that results in their constructive interference and therefore transient appears to start from the maximum of the first beat. Thus, the minimum length of acquisition required to acquire two beats and achieve even relatively poor resolution of the isotopes, can be estimated as $\left(\Delta t_{i}+\right.$ $\left.\Delta t_{w}\right)$. For proteins with mass of several tens $\mathrm{kDa}$ and typical experimental parameters, this value lies in the range from few hundreds of $\mathrm{ms}$ to $1 \mathrm{~s}$.

\section{Decay of Transients of Protein Ions in the Orbitrap Mass Analyzer}

In Orbitrap-based mass spectrometers, ions are characterized by much higher values of kinetic energy (1.5 to $3.6 \mathrm{keV} /$ charge) which, unlike those in FT ICR instruments, are also independent of mass-to-charge ratio $m / z$. This typically results in higher center-of-mass collision energies and hence much faster signal decay than in FT ICR at comparable residual gas pressures. As a result, the amplitude of beats is also decreasing with time. The evolution of a transient envelope with time for a particular $\mathrm{m} / \mathrm{z}$ can be described by a general formula:

$$
A(t)=A_{0} \cdot C(t) \cdot S(t)
$$

where $A_{0}$ corresponds to the intensity at $t=0$ (roughly equal to the amplitude of the first bit), $C(t)$ is the term describing loss of ions of that $\mathrm{m} / \mathrm{z}$ in collisions (fragmenting collisions) and $S(t)$ is the term describing reduction of signal caused by all other effects such as:

1. Dephasing of ion packet due to manufacturing imperfections.

2. Dephasing of ion packet due to scattering on nonfragmenting collisions.

3. Reduction of radial and axial amplitudes of oscillations due to nonfragmenting collisions (friction).

The first effect, dephasing caused by manufacturing imperfections, is typically not important for unscattered protein ions but becomes troublesome when combined with the third effect. If protein ions lose so much energy that they descend into an orbit very near the central electrode (e.g., 1-2 mm away), short-range fields of local manufacturing imperfections will accelerate dephasing greatly. In addition, the central electrode would absorb a rapidly increasing share of the image current, thus further reducing the detected signal. This would result in disappearance of the detected signal even though ions are still physically present. A similar effect could be caused by the second effect, which is always combined with the third one.

Loss of ions in collisions with background gas is a strictly statistical process and therefore could be described as [7]:

$$
C(t)=\exp (-c \cdot t)
$$

where $c$ is a constant linked to the collision cross-section $\sigma_{\text {coll }}$, concentration of background gas $n$ and relative velocity $v$ :

$$
c=n \cdot \sigma_{\text {coll }} \cdot \nu
$$

At high ion energies typical for the Orbitrap analyzer, the relative velocity $v$ is approximately equal to the ion speed. Therefore, 


$$
\nu \approx \sqrt{\frac{2 e V}{(m / z)}}
$$

where $z e V$ is the final ion energy inside the trap.

Term $S(t)$ in eq 7 cannot be expected a priori to follow an exponential law. However, extensive analyses of transients for various protein ions (such as those in Figure 1) have shown that the formula 7 is well approximated by an exponential law:

$$
A(t)=A_{0} \exp (-\lambda \cdot t)
$$

where $\lambda$ is a constant, $\lambda=c+s$ with $s$ coming from the resulting approximation:

$$
S(t) \approx \exp (-s \cdot t)
$$

While constant $\lambda$ could be easily deduced from experimental transients, it would be very difficult to deduce each of the constants $c$ and $s$. However, they can be approximately estimated if we eliminate effects linked to the dephasing of ion packets. This can be achieved by acquiring transients not from packets containing multi-

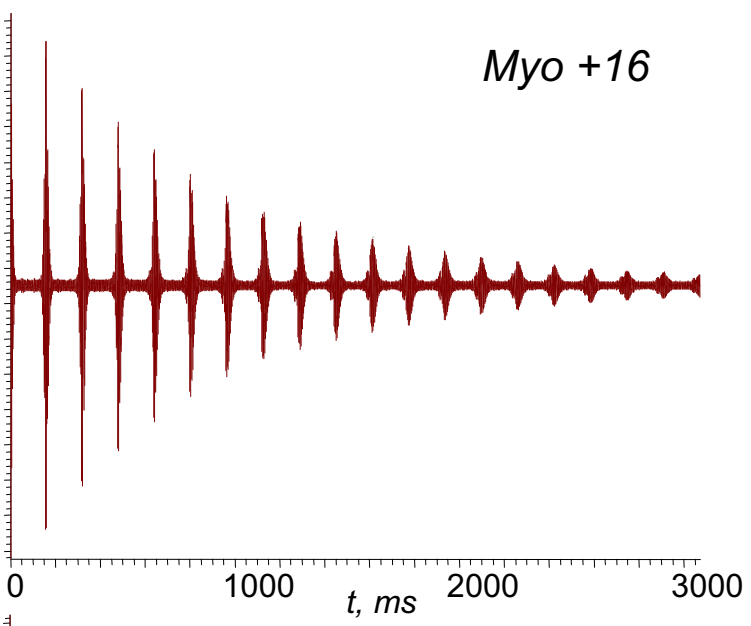

(a)

$t, m$

000

(c) 0

$1000 t, m s \quad 2000 \quad 3000$ ple ions but from individual ions [8]. This became the motivation for the second part of this work: detection of individual ions using Orbitrap analyzer.

Constant $\lambda$ is linked to the decay constant $\tau$ of transient (i.e., time duration over which transient amplitude decays by $e$ times) as $\lambda=1 / \tau$. Similarly to (9-10), this experimentally measured $\tau$ could be used to calculate the decay length $L=1 / n \sigma$ directly linked to the total cross-section $\sigma$ of an ion:

$$
L=\frac{1}{n \sigma}=\nu \cdot \tau=\sqrt{\frac{2 e V}{(m / z)}} \cdot \tau
$$

To resolve the isotopic envelope of proteins, the Orbitrap vacuum must be improved to such an extent that constant $\lambda$ is long enough to acquire two or more beats. In addition, the fragmentation of protein ions must be avoided throughout their entire path towards the Orbitrap analyzer.

\section{Experimental}

Using a standard LTQ Orbitrap XL mass spectrometer $[5,9]$ as a test-bed, higher power bake-out heaters were
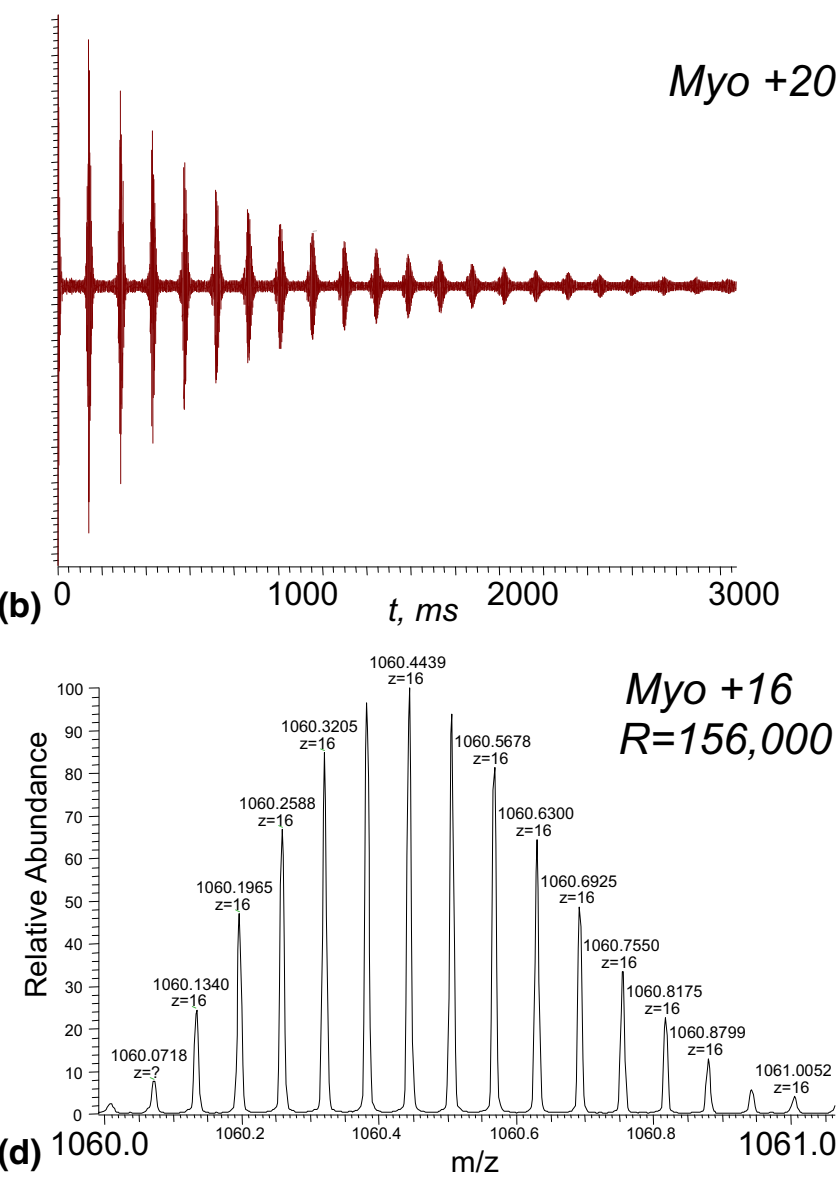

Figure 1. Examples of transients for different charge states of horse heart myoglobin protein $(\mathbf{a})+16$, $(\mathbf{b})+20$, (c) +22 ). Transient (a) results in a mass spectrum (d), shown with average resolving power for this charge state. 
employed to raise bake-out temperature from the standard $110^{\circ} \mathrm{C}$ to $180^{\circ} \mathrm{C}$. This allowed pressure in the Orbitrap compartment to be reduced below $10^{-10} \mathrm{mbar}$ level following just a 12-h bake-out after venting and ultimately down to $5 \times 10^{-11} \mathrm{mbar}$. This ultimate pressure remained then at that level until the next venting of the instrument. A standard preamplifier and electronics were used.

To reduce protein fragmentation near the exit of the C-trap as well as gas flow to the Orbitrap compartment, the gas line to HCD cell was blocked. Background gas in the linear trap compartment consisted of helium from the linear trap (partial uncorrected pressure $10^{-5} \mathrm{mbar}$ as indicated by hot-cathode ion gauge), nitrogen from the C-trap (partial pressure $0.4 \times 10^{-5} \mathrm{mbar}$ ) and nitrogen flowing from the electrospray source (partial pressure $0.25 \times 10^{-5}$ mbar). Nitrogen from the C-trap was the main outside contributor to the background gas in the Orbitrap compartment. Its partial pressure was around $1 \times 10^{-11} \mathrm{mbar}$ as measured by a cold-cathode ion gauge near $300 \mathrm{~L} / \mathrm{s}$ turbopump. Using residual gas analyzer QMG 220 M3 (Pfeiffer, Asslar, Germany), it was found that the remaining background gas in the vacuum chamber was hydrogen, presumably diffusing through the stainless steel walls of the vacuum chamber. Nevertheless, gas in the immediate vicinity and inside the Orbitrap is expected to be mainly nitrogen at pressures at least 3-4 times higher than the indicated above.

The instrument was calibrated using the standard LTQ calibration mixture with caffeine, peptide MRFA, and Ultramark 1600 dissolved in 25:25:49:1 vol.vol water/methanol/acetonitrile/acetic acid solution. Proteins such as horse heart myoglobin (16.9 kDa), carbonic anhydrase (29 kDa), yeast enolase (46.6 kDa), bovine serum albumin (66 kDa), and bovine apo-transferrin (78 $\mathrm{kDa}$ ) were obtained from Sigma-Aldrich Chemie $\mathrm{GmbH}$ (Munich, Germany) and used without further purification. Protein solutions were infused in a static mode using the IonMax electrospray source, with a charge state selected by the LTQ mass spectrometer, until a predefined number of ions was reached. The resulting ion population was transferred into the nitrogen-filled C-trap, trapped, and then ejected towards the entrance aperture of the Orbitrap. An acquisition time of $3 \mathrm{~s}$ was used for detection of protein transients. It is important to note that all experiments were carried out with external calibration.

Unless stated otherwise, Fourier transform of transients was performed using a single zero-filling and Kaiser-Bessel apodization, with the frequency spectrum converted into a mass spectrum using a two-point calibration. Mass spectral data were stored in fullprofile or reduced-profile format and processed with
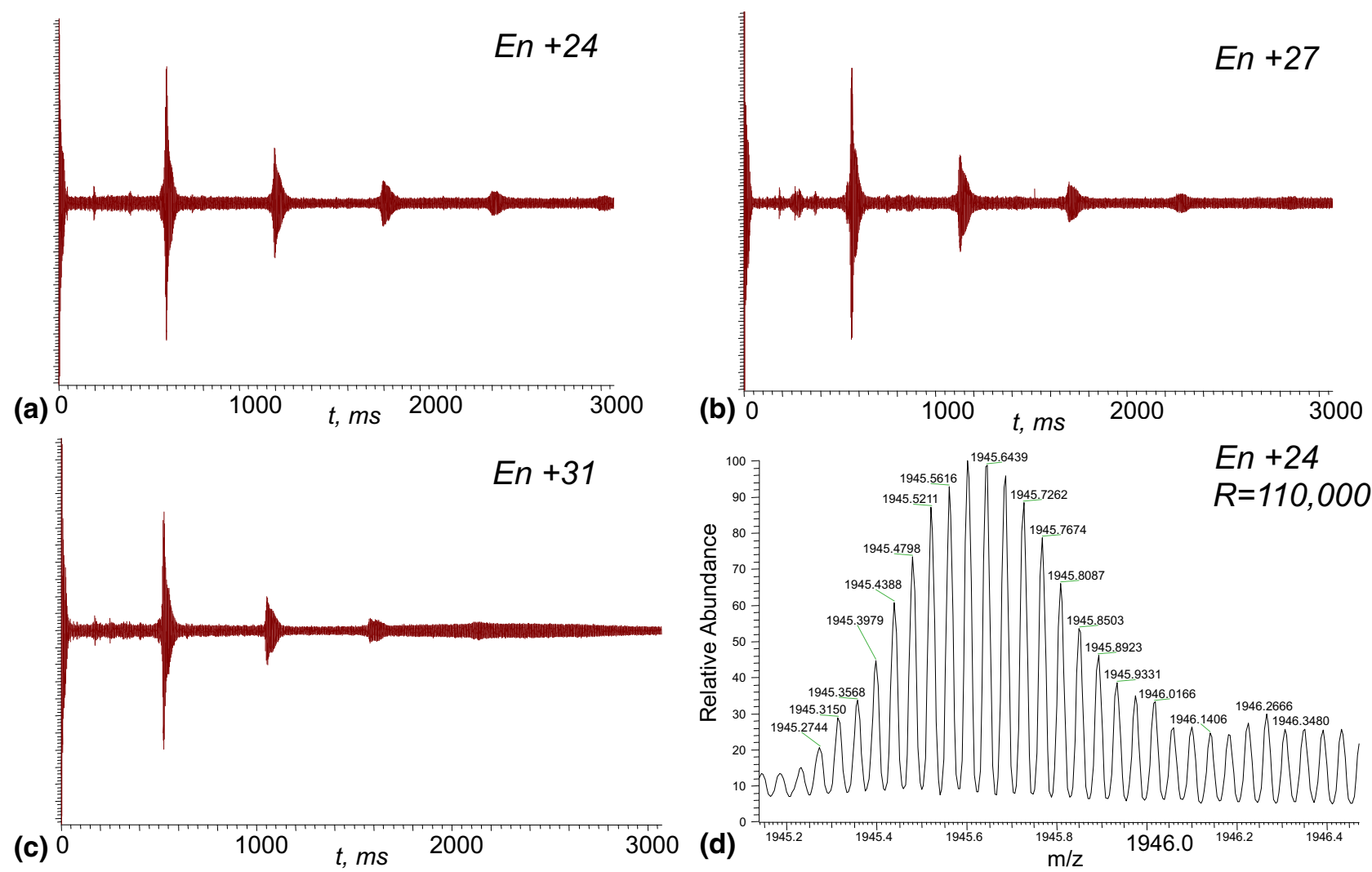

Figure 2. Examples of transients for different charge states of enolase protein (a) $+24,(\mathbf{b})+27$, (c) +31 ). Transient (a) results in a mass spectrum shown in (d) with average resolving power for this charge state. 
Xcalibur software. Apodization was switched off when only a few beats in transients were observed.

\section{Results and Discussion}

Reduction of background pressure inside the Orbitrap compartment has indeed enabled drastic deceleration of signal decay and significantly extended the limit for the highest-mass isotopically-resolved proteins. Examples of transients for some proteins as well as corresponding isotopic distributions are presented in Figures 1-3. Figure 3 shows isotopic distributions for BSA (66.4 $\mathrm{kDa})$ and transferrin $(78 \mathrm{kDa})$, so far the heaviest proteins isotopically resolved using the Orbitrap mass analyzer.
A comparison of the transients for different charge states of myoglobin in Figure 1 and enolase in Figure 2 immediately uncovers substantial differences in their decay times. This effect is summarized for different proteins in Table 1 for final average kinetic energy of about $1500 \mathrm{~V}$ per charge.

Several observations can be made from this table. First, there is a monotonous and quite steep increase of the cross-section as charge state increases. This increase is typically steeper than the increase of cross-sections previously observed in ion mobility experiments, e.g., for myoglobin ions $[10,11]$. Therefore, the main reason for the additional growth of measured cross-section is likely to be the increase of center-of-mass collision energy (proportional to $(\mathrm{m} / \mathrm{z}) / \mathrm{m}_{\mathrm{g}}$, where $m_{g}$ is the mass
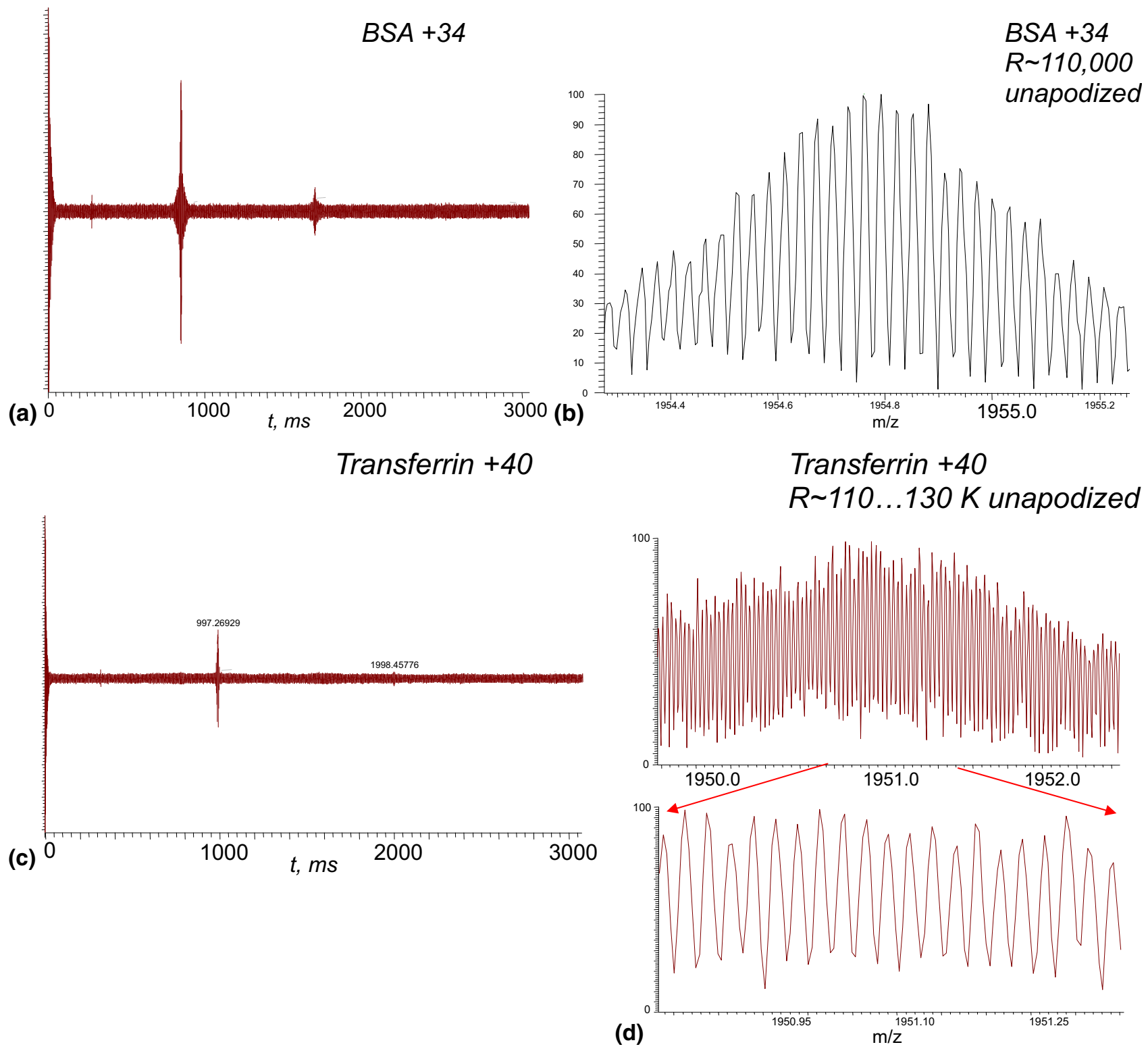

Figure 3. Examples of transients for +34 charge state of bovine serum albumin (a) and +40 charge state of transferrin (c) together with resulting mass spectra $(\mathbf{b})$ and (d), respectively. No apodization was employed with Fourier transform. 
Table 1. Overview of experimentally estimated transient decay time $T$, corresponding decay length $L$ and relative total cross-section $\sigma$

\begin{tabular}{lcccccc}
\hline \multicolumn{1}{c}{ Protein } & $\mathrm{M}, \mathrm{kDa}$ & $z$ & Avg $\mathrm{m} / \mathrm{z}$ & $\tau, \mathrm{s}$ & $\mathrm{L}, \mathrm{m}$ & Rel. total cross-section \\
\hline \hline Myoglobin & 16.9 & 16 & 1060.4 & 0.82 & $1.4 \mathrm{E}+04$ & 0.85 \\
& & 20 & 848.6 & 0.62 & $1.2 \mathrm{E}+04$ & 1 \\
Carbonic anhydrase & \multirow{2}{*}{29} & 22 & 771.5 & 0.48 & $9.5 \mathrm{E}+03$ & 1.23 \\
& & 20 & 1452.2 & 0.65 & $9.3 \mathrm{E}+03$ & 1.25 \\
Enolase & & 24 & 1210.4 & 0.49 & $7.7 \mathrm{E}+03$ & 1.51 \\
& \multirow{2}{*}{46.6} & 26 & 1117.4 & 0.48 & $7.9 \mathrm{E}+03$ & 1.48 \\
BSA & & 24 & 1945.6 & 0.62 & $7.7 \mathrm{E}+03$ & 1.51 \\
Transferrin & \multirow{2}{*}{66.4} & 31 & 1729.5 & 0.51 & $6.7 \mathrm{E}+03$ & 1.74 \\
\hline
\end{tabular}

number for residual gas) and hence energy deposition into the protein ion. It can also be observed that the cross-section is not a monotonous function of $\mathrm{M}$ even for a similar $\mathrm{m} / \mathrm{z}$ of different proteins. This might originate from the difference in conformation-dependant dissociation thresholds.

Unfortunately, there is no current provision to measure pressure inside the Orbitrap analyzer directly,

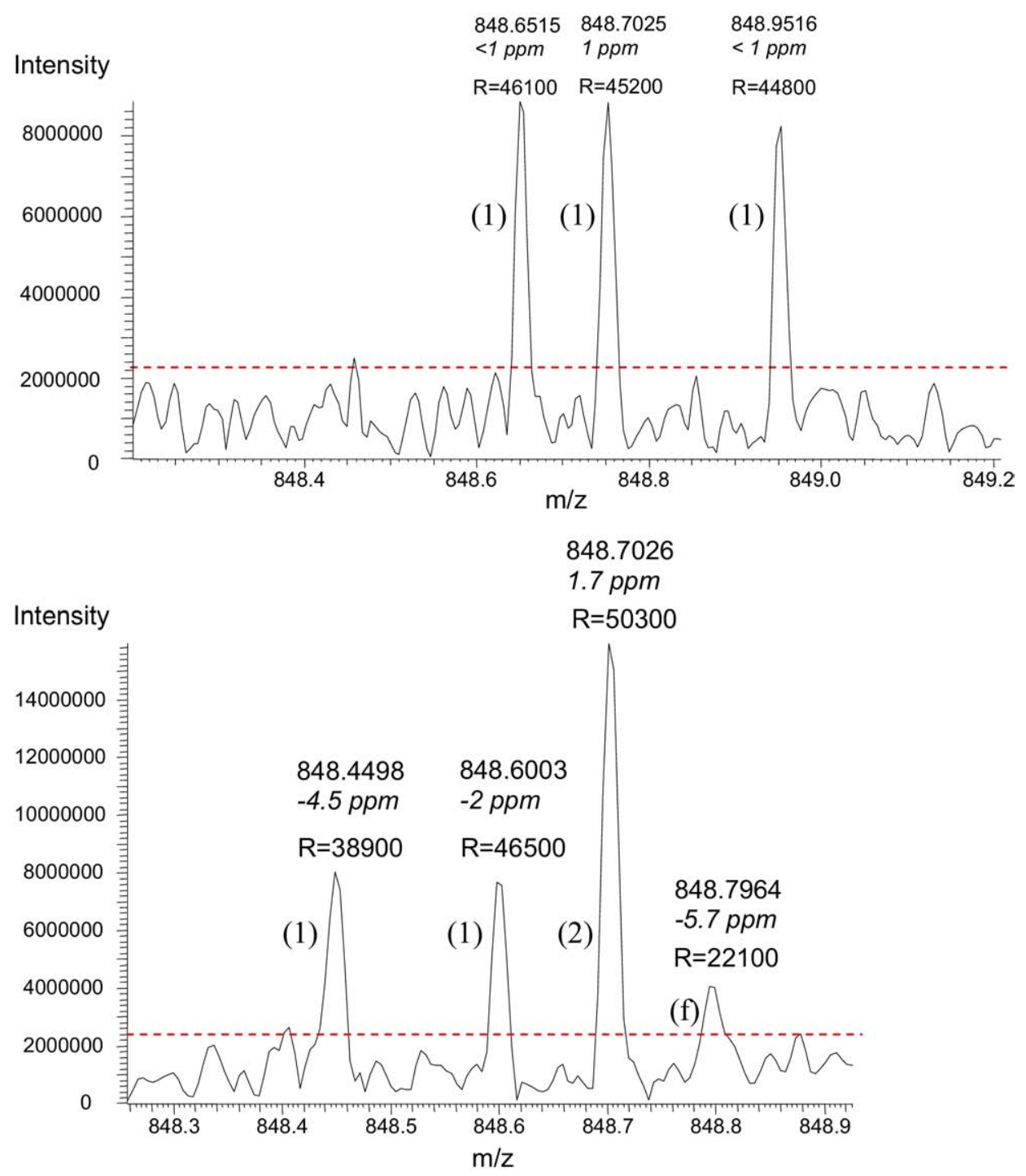

Figure 4. Examples of spectra of individual ions. Single ions are denoted as (1), two individual ions with the same $\mathrm{m} / \mathrm{z}$ as (2), and (f) denotes a single ion that is likely to have fragmented during the transient. Dashed line indicates $\mathrm{S} / \mathrm{N}=1$ as determined by Xcalibur. 
therefore only order-of-magnitude estimations can be made for the concentration $n$ of collision gas. These estimations result in cross-section values that are comparable to those in [10] but significantly greater than in [12]. For this reason, Table 1 contains only crosssections relative to the +20 myoglobin protein ion that allows exclusion of this uncertainty and limits the error to about $5 \%$ to $10 \%$. This approach can generally be used to investigate conformation-related differences in protein cross-sections.

An attempt was also made to estimate the effects linked to the de-phasing of ion packets (i.e., approximately estimate constants $c$ and $s$ in eq 8 and eq 12). This was achieved by detecting individual ions of +20 charge state of myoglobin as shown in Figure 4 . The +20 charge state was isolated in the linear trap and then transferred via the C-trap into the Orbitrap mass analyzer. After acquiring a conventional spectrum for packets of 10,000 ions as a reference, ion filling time was reduced until only 1 to 2 individual +20 ions per spectrum were delivered to the Orbitrap. Spectra were acquired at nominal resolving powers $60,000(46,000$ at $\mathrm{m} / \mathrm{z} 848$ of +20 charge state, $0.76 \mathrm{~s}$ acquisition time) and 100,000 (92,000 at $\mathrm{m} / \mathrm{z} 848$ of +20 charge state, $1.52 \mathrm{~s}$ acquisition time). The noise band was automatically calculated by Xcalibur for the thermal noise of the pre-amplifier and corresponded to $\sim 5-6$ r.m.s. deviations of this noise. Peaks at $S / N=1$ or below are not detected as peaks.

Detection of individual ions can only be proven if the intensity of peaks in Figure 4 follows a quantized

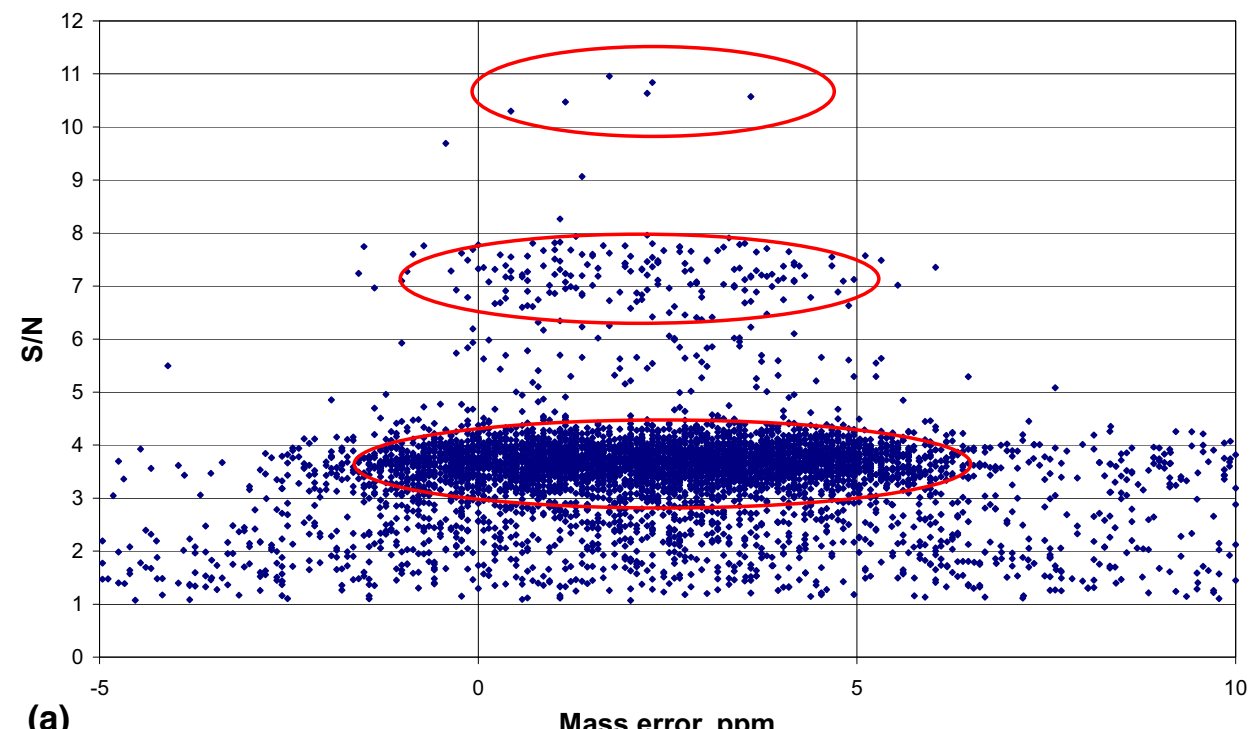

(a)

Mass error, ppm

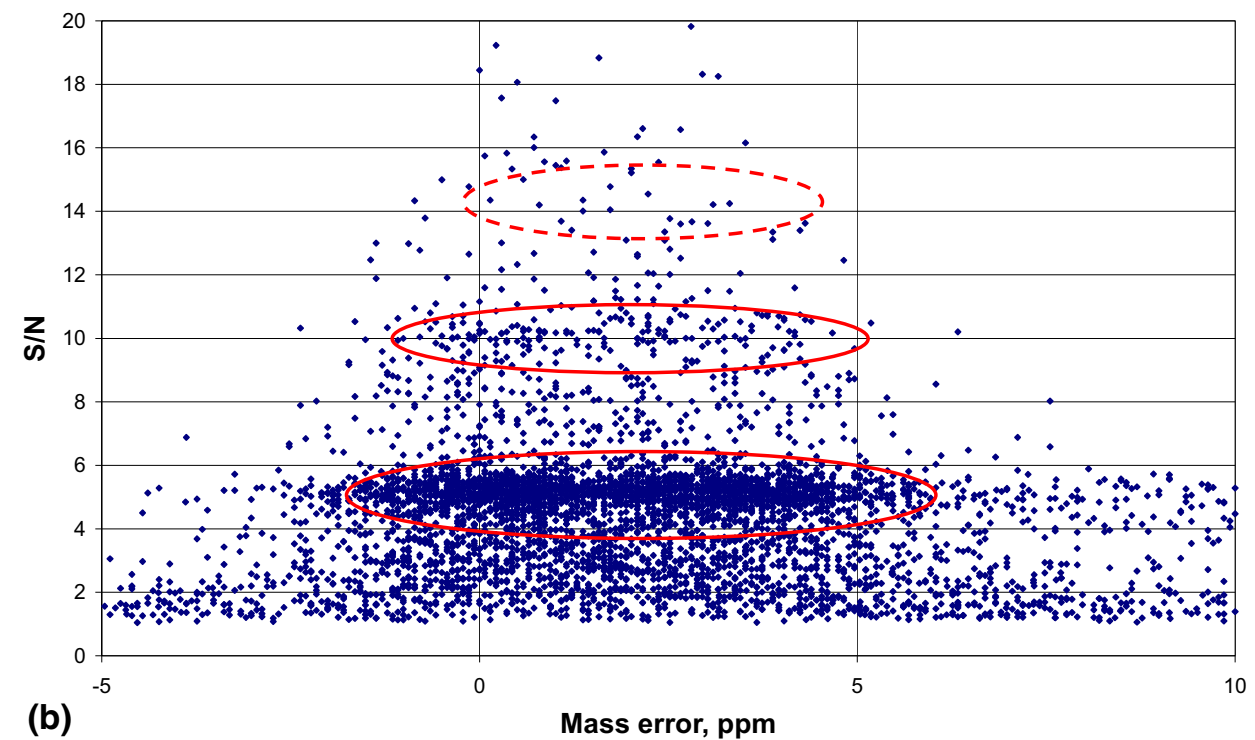

Figure 5. Distribution of detected peaks on their mass difference relatively to their nearest isotopomers of myoglobin +20 and on $\mathrm{S} / \mathrm{N}$ for (a) acquisition time $0.76 \mathrm{~s}$ (nominal resolving power 60,000); (b) acquisition time $1.52 \mathrm{~s}$ (nominal resolving power 100,000). Areas of high peak concentration are indicated by ellipses. 
distribution of signal-to-noise $(\mathrm{S} / \mathrm{N})$ ratios, i.e., $\mathrm{S} / \mathrm{N}$ should change in quantum steps as 1,2 , 3, etc. [8]. Analysis of thousands of spectra not only confirmed such quantization, but also allowed grouping of detected ions according to the error of measured $\mathrm{m} / \mathrm{z}$ relative to the nearest isotopic peak of myoglobin (Figure 5). In this case low mass error and appropriate resolving power (around 46,000 and 92,000 for 0.76 and $1.52 \mathrm{~s}$ acquisitions, respectively) independently prove that the detected signal indeed came from an ion that survived until the end of the acquisition. Figure 5a shows clearly three consecutive equidistant "islands" with progressively lower ion populations as well as a background that is almost uniformly distributed along $\mathrm{m} / \mathrm{z}$ axis, mostly below $\mathrm{S} / \mathrm{N}=2.5$. These islands (espe- cially the first one) are clearly visible also on a histogram presented in Figure $6 \mathrm{a}$ and allow to calculate average signal-to-noise ratio $\mathrm{S} / \mathrm{N}$ at which a single ion or simultaneously injected 2 to 3 ions of the same $\mathrm{m} / \mathrm{z}$ could be measured. For example, a single +20 ion is measured in $0.76 \mathrm{~s}$ acquisition with $\mathrm{S} / \mathrm{N}=3.6 \pm 0.4$ (r.m.s.), for double-S/N $=7.2 \pm 0.4$ (r.m.s.), for triple$\mathrm{S} / \mathrm{N}=10.5 \pm 0.4$ (r.m.s.). In a $1.52 \mathrm{~s}$ acquisition, the signal of surviving ions was found to remain practically unchanged while noise band decreased as a square-root of acquisition time, i.e., by a factor of $2^{1 / 2}$ (Figure $5 b$ ). As a result, for a single +20 ion we have measured $\mathrm{S} / \mathrm{N}=$ $5.1 \pm 0.4$ (r.m.s.), for double-S/N = $10.2 \pm 0.5$ (r.m.s.), for triple-S/N $=15.3 \pm 0.9$ (r.m.s.). The increased diffuse background in Figure $5 \mathrm{~b}$ clearly demonstrates a
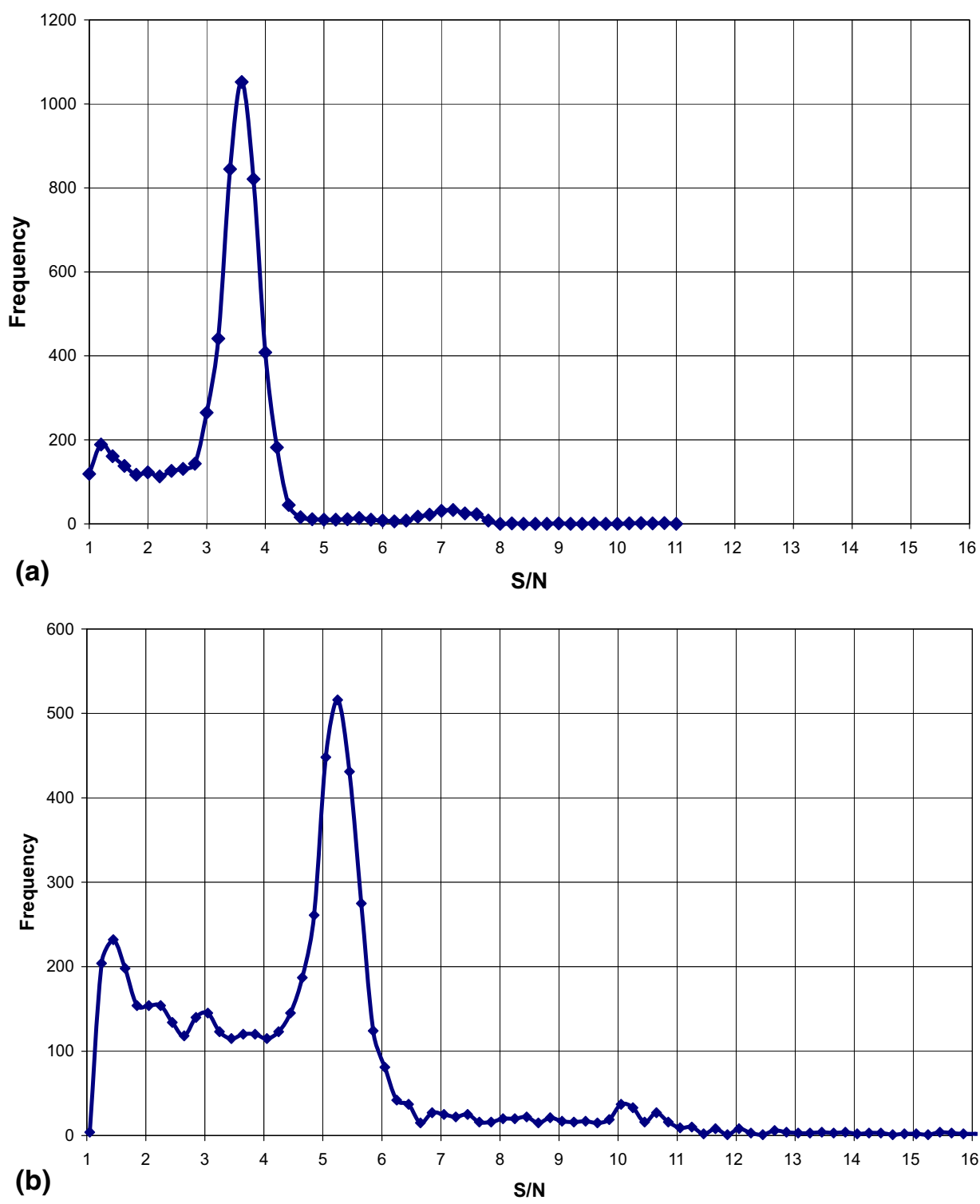

Figure 6. The graph shows the frequency of detected myoglobin +20 peaks for different $\mathrm{S} / \mathrm{N}$ (histogram) for bins of 0.2 width for (a) acquisition time $0.76 \mathrm{~s}$, around 5700 peaks total; (b) acquisition time $1.52 \mathrm{~s}$, around 5400 peaks total. Note the increased left shoulder of the first peak in (b) indicating increased loss of individual ions. 
decreased probability for an ion to survive until the end of the transient, progressively reducing for two or more ions of the same $m / z$ simultaneously injected in the trap. It is interesting to note that even single ions with $\mathrm{S} / \mathrm{N}=$ 3-5 exhibit r.m.s. spread of measured $\mathrm{m} / \mathrm{z}$ below $3 \mathrm{ppm}$ and it drops to 1-1.7 ppm for $\mathrm{S} / \mathrm{N}>7$ (systematic shift of $2 \mathrm{ppm}$ is just the result of external calibration). This fits with observations from [13].

These data prove that the full noise band of a standard Orbitrap preamplifier for a $0.76 \mathrm{~s}$ acquisition is equivalent to about five to six elementary charges, whilst the full noise band for a $1.5 \mathrm{~s}$ acquisition is equivalent to about four elementary charges! This brings image current detection much closer to the single-charge detection than previously commonly believed by mass spectrometrists. This also fits with the r.m.s. noise figure of $0.8-1 \mathrm{nV} / \mathrm{Hz}^{1 / 2}$ quoted by the supplier of field-emission transistors for the standard Orbitrap pre-amplifier.

The peaks outside of the "islands" in Figure 5 typically exhibit lower resolution, indicating premature disappearance of corresponding individual ions from the entire $1 \mathrm{Da}$ window. These ions are expected to be lost due to fragmentation and therefore they can be used to estimate the corresponding signal decay process. By choosing a diffuse band of $\mathrm{S} / \mathrm{N}$ above 1 but clearly below the first peak on Figure 6 (e.g., S/N range from 1.2 to 2.6 in Figure $6 a$ and 1.2 to 4 in Figure $6 \mathrm{~b}$ ), we can relate the number of counts in this diffuse band to the total number of surviving ions within \pm 2 r.m.s. from the mean value for the first peak. It should be noted that this proportion could serve only as an upper estimation of lost ions as some counts, especially at $\mathrm{S} / \mathrm{N}=$ 1.2 or below, could be just rare outliers of electronic noise. Assuming approximate proportionality of detected signal from "lost" ions to their lifetime in the trap, we can estimate the start and the end of the time interval when an ion should fragment to be registered in this diffuse band. The numerical solution of eq 8 then yields $c \approx 0.65-0.72 \mathrm{~s}^{-1}$ (the lower value for Figure $6 \mathrm{~b}$ and the higher for 6a). This corresponds to the decay constant due to fragmentation of $1 / c=1.35-1.55 \mathrm{~s}$. This, in turn, differs significantly from $\tau \approx 0.62 \mathrm{~s}$ in Table 1 and allows for the estimation of $s$ in eq 12 as $s \approx$ 0.9-0.97 $\mathrm{s}^{-1}$ and the corresponding decay constant due to dephasing as $1 / \mathrm{s}=1-1.1 \mathrm{~s}$. Therefore we conclude that loss due to fragmentation is responsible for transient decay to approximately the same extent as scattering and dephasing together and none of the factors dominate absolutely.

A detailed comparison of absolute signal amplitudes for surviving single ions in 0.76 and $1.52 \mathrm{~s}$ acquisitions also reveals no difference within the experimental error of measurement. This means that reduction of radial and axial amplitudes of oscillations due to nonfragmenting collisions (friction) is negligible, thus only fragmentation and dephasing are responsible for signal decay. Unspecific low-intensity fragments observed both for individual ions and for ion packets are de- scribed in Supplemental Information, which can be found in the electronic version of this article.

\section{Conclusions}

After a good residual vacuum is established, the Orbitrap analyzer can then be used for analysis of intact proteins of small to medium size with isotopic resolution. The ability to select a protein charge state in the linear trap opens the possibility to investigate decay curves for proteins of each charge state separately and therefore calculate relative cross-sections. Deviations in protein cross-sections for a given $\mathrm{m} / \mathrm{z}$ are expected to reflect conformational information and form a subject of future research.

The possibility of achieving individual ion detection in the Orbitrap mass analyzer was proven by observing the quantized distribution of signal intensities for +20 myoglobin ions well above the noise threshold, with high mass accuracy and resolution of recorded individual ions being used as independent confirmation of correct assignment of signal to ions rather than to noise. These experiments demonstrated that the measured decay of transients for protein ions is a consequence of two major factors of approximately equal importance: loss of ions in fragmenting collisions and dephasing of ion packets due to scattering. Change of ion orbits due to friction was shown to be negligible.

\section{Acknowledgments}

The authors thank Dr. Jens Griep-Raming, Dr. Vlad Zabrouskov, Dr. Lester Taylor, and Dr. Stevan Horning for support and fruitful discussions, Dr. Kerstin Strupat and Dr. Eugene Damoc for help with preparing protein samples. The research leading to these results has received funding from the European Commission's 7th Framework Programme (grant agreement HEALTH-F4-2008201648/PROSPECTS).

\section{Appendix A Supplementary Material}

Supplementary material associated with this article may be found in the online version at doi:10.1016/ j.jasms.2009.03.024.

\section{References}

1. Marshall, A. G.; Hendrickson, C. L.; Jackson, G. S. Fourier Transform Ion Cyclotron Resonance Mass Spectrometry: A Primer. Mass Spectrom. Rev. 1998, 17, 1-35.

2. Ge, Y.; Xu, L.; Rybakova, I.; Zabrouskov, V.; Moss, R. L.; Walker, J. W. Top Down Disease Proteomics: Deciphering Protein Modifications for Understanding and Diagnosis of Human Diseases. Proceedings of the 56th ASMS Conference on Mass Spectrometry; Denver, CO, June, 2008.

3. Hu, Q.; Li, H.; Makarov, A.; Cooks, R. G.; Noll, R. J. Resonant AC Dipolar Excitation for Ion Motion Control in the Orbitrap Mass Analyzer. J. Phys. Chem. A 2006, 110, 2682-2689.

4. Bondarenko, P. V.; Second, T. P.; Zabrouskov, V.; Makarov, A.; Zhang Z. Intact Mass and Top-Down HPLC/MS Analysis of Monoclonal IgG Antibodies on Orbitrap. J. Am. Soc. Mass Spectrom. 2009, this issue.

5. Makarov, A.; Denisov, E.; Kholomeev, A.; Balschun, W.; Lange, O.; Horning, S.; Strupat, K. Performance Evaluation of a Hybrid Linear Ion Trap/Orbitrap Mass Spectrometer. Anal. Chem. 2006, 78, 2113-2120. 
6. Hofstadler, S. A.; Bruce, J. E.; Rockwood, A. L.; Anderson, G. A.; Winger, B. E.; Smith, R. D. Isotopic Beat Patterns in Fourier Transform Ion Cyclotron Resonance Mass Spectrometry: Implications for High Resolution Mass Measurements of Large Biopolymers. Int. J. Mass Spectrom. Ion Processes 1994, 132, 109-127.

7. Spengler, B.; Kirsch, D.; Kaufmann, R. Fundamental Aspects of Postsource Decay in Matrix-Assisted Laser Desorption Mass Spectrometry. 1. Residual Gas Effects. J. Phys. Chem. 1992, 96, 9678-9684.

8. (a) Bruce, J. E.; Cheng, X.; Bakhtiar, R.; Wu, Q.; Hofstadler, S. A.; Anderson, G. A.; Smith, R. D. Trapping, Detection, and Mass Measurement of Individual Ions in a Fourier Transform Ion Cyclotron Resonance Mass Spectrometer. J. Am. Chem. Soc. Mass Spectrom. 1994, 116, 1839-1841. (b) Cheng, X.; Bakhtiar, R.; Van Orden, S.; Smith, R. D. Charge-State Shifting of Individual Multiply-Charged Ions of Bovine Albumin Dimer and Molecular Weight Determination Using an Individual Ion Approach. Anal. Chem. 1994, 66, 2084-2087. (c) Smith, R. D.; Cheng, X.; Bruce, J. E.; Hofstadler, S. A.; Anderson, G. A. Trapping,
Detection, and Reaction of Very Large Single Molecular Ions by Mass Spectrometry. Nature 1994, 369, 137-139.

9. Olsen, J. V.; Macek, B.; Lange, O.; Makarov, A.; Horning, S.; Mann, M. Higher-Energy C-Trap Dissociation for Peptide Modification Analysis. Nat. Methods 2007, 4, 709-712.

10. Covey, T.; Douglas, D. J. Collision Cross Sections for Protein Ions. J. Am. Soc. Mass Spectrom. 1993, 4, 616-623.

11. Javahery, G.; Thomson, B. A Segmented Radiofrequency-Only Quadrupole Collision Cell for Measurements of Ion Collision Cross Section on a Triple Quadrupole Mass Spectrometer. J. Am. Soc. Mass Spectrom. 1997, 8, 697-702.

12. Chernushevich, I. V.; Verentchikov, A. N.; Ens, W.; Standing, K. G. Effect of Ion-Molecule Collisions in the Vacuum Chamber of an Electrospray Time-of-Flight Mass Spectrometer on Mass Spectra of Proteins. J. Am. Soc. Mass Spectrom. 1996, 7, 342-349.

13. Makarov, A.; Denisov, E.; Lange, O.; Horning, S. Dynamic Range of Mass Accuracy in LTQ Orbitrap Hybrid Mass Spectrometer. J. Am. Soc. Mass Spectrom. 2006, 17, 977-982. 